I.

Aus der medizinischen Universitätsklinik in Kiel.

Klinische und experimentelle Beobachtnugen über Benzinvergiftung.

Von

Prof. A. Böhme, Kiel und Dr. R. Köster, Flensburg, früher Kiel.

Fälle von Vergiftung mit Benzin - einem Destillationsprodult des Petroleums, das in der Hauptsache ans Hexan $\mathrm{C}_{6} \mathrm{H}_{8}$ und Heptan $\mathrm{C}_{7} \mathrm{H}_{14}$ besteht - sind nicht sehr häufig. Sie ereignen sich im allgemeinen entweder durch Einatmung von Benzindämpfen in gewerblichen Betrieben, in denen mit Benzin gearbeitet wird, oder durch Trinken von Benzin, das meist aus Versehen, nur selten zum Zwecke des Selbstmordes genommen wird. Die überwiegende Mehrzahl der durch Trinken von Benzin entstandenen Vergiftungen betrifft Kinder und hat bei diesen meist rasch zum Tode geführt. Die durch Trinken entstandenen Vergiftungen der Erwachsenen sind demgegenüber meist leichter verlaufen.

Die Vergiftung durch Inhalation von Benzin führt sehr rasch zu Ohnmacht und tiefer Bewaßtlosigkeit, Zyanose, Reflexstörungen, gelegentlich Krämpfen und Lähmungen (Foulerton ${ }^{1}$ ), Si emon ${ }^{2}$ ), Burgi ${ }^{3}$ ), Wichern ${ }^{4}$ )), also zu einer auf Vergiftung des Zentralnervensystems beruhenden tiefen Narkose. Wird der Erkrankte rasch genug aus den Benzindämpfen entfernt, so tritt meist baldige Erholung ein. Es können unter Umständen noch später länger dauernde Schädigungen des Zentralnervensystems sich einstellen (Peters 5 ), Neuritis retrobulbaris, Dorner ${ }^{6}$ ), Rückenmarkserkrankung).

1) Lancet 1886, S. 865 .

2) Monatsschr. f. Unfallheilk. 1896, Bd. III, S. 366.

3) Korrespondenzblatt f. Schweizer Ärzte 1906, Bd. 36, S. 11.

4) Münch. med. Wochenschr. 1909, Nr. 1.

5) Deutsch. med. Wochenschr. 1900, Vereinsbeil., S. 249.

6) Dentsche Zeitschr. f. Nervenheilk. 1915. 
In den - meist bei Kindern beobachteten - Fällen tödlicher oraler Vergiftung kam es ebenfalls rasch zu Bewußtlosigkeit, Zyanose und Kälte der Haut, Weite und Reaktionslosigkeit der Pupillen. Der Tod trat schon nach wenigen Minuten oder doch innerhalb einiger Stunden ein. Die Autopsie ergab häufig Lungenblutungen (Racin ${ }^{1}$ ), Burgl ${ }^{2}$ ), Klare ${ }^{3}$ ), Jaffé $\left.{ }^{4}\right)$ ). In einigen Fällen längerer Dauer bei Erwachsenen kam es zu Pneumonie oder putrider Bronchitis. Meist wurden leichte Veränderungen der Schleimhaut des Verdauungstraktus (Hyperämie, kleine Blutungen), ferner leichte Nierenveränderungen mit Albuminurie gefunden. Racine und Burgl beobachteten außerdem hämolytische Veränderungen. In anderen Fällen (Falk ${ }^{5}$ ), Rosentha ${ }^{6}$ )) wird der Sektionsbefund als negativ bezeichnet. Jedenfalls ist sowohl das klinische wie das anatomische Bild der oralen Vergiftung aus einer ganzen Reihe verschiedenartiger Veränderungen zusammengesetzt. Während bei Kindern der Tod meist schon nach Einnahme von etwa $10-15 \mathrm{~g}$ eintrat, vertragen Erwachsene oft viel größere Dosen, ohne schwerere Vergiftungserscheinungen zu bekommen. In Fall 3 von Wichern war selbst nach Einnahme von $1 / 41$ Benzin nur zeitweise Bewußtlosigkeit und danach völlige Wiederherstellung eingetreten.

Zwei in der medizinischen Klinik in Kiel in den Jahren 1912 und 1913 beobachtete Fälle von oraler Benzinvergiftung, die ebenfalls diesen Unterschied in den Symptomen zwischen Kind und Erwachsenem zeigen, mögen zur Kenntnis des Krankheitsbildes beitragen.

\section{Fall 1.}

2 jähriges Kind, hat mittags gegen 12 Uhr aus einer auf dem Tisch stehenden Flasche Benzin getrunken. Die Menge kann nach dem noch in der Flasche verbliebenen Rest nur gering gewesen sein, etwa $7 \mathrm{ccm}$. Das Kind schrie auf, gleich nachdem es getrunken hatte, hustete und spie etwas blutige Flüssigkeit aus. Erbrechen trat zunächst nicht ein. Die Mutter brachte das Kind sofort nach der Klinik. Das kräftig entwickelte, gut genährte Kind ist unruhig, reagiert dabei nicht auf Anruf. Hant sehr blaß und kalt, am Rumpf bläulich-weiß marmoriert, an den Extremitäten und dem Gesicht stark zyanotisch. Im Rachen blutige schaumige Massen. Dys- und Tachypnöe. 38 A temzüge in der Minute. Das Kind stöhnt bei jeder Exspiration. Verschärftes Atemgeräusch, Herztöne rein, sehr beschleunigt, 200 in der Minute, régelmäßig. Puls kaum fühlbar. Abdomen

1) Vierteljahrschr. f. ger. Med. 1901, Bd. 22.

2) Mïnch. med. Wochenschr. 1906, S. 412.

3) Ärztl. Sachverständ.-Zeitung 1907, S. 93.

4) Münch. med. Wochenschr. 1914, S. 175.

5) Vierteljahrsschr. f. ger. Med. 1892, Bd. 3.

6) Zentralblatt f. inn. Med. 1894, S. 281. 
etwas aufgetrieben. Epigastrium druckempfindlich. Muskelwiderstand beim Betasten. Pupillen von mittlerer und gleicher Weite, Lichtreaktion vorhanden. Temperatur 37,4 ${ }^{\circ}$. Die sofort nach der Aufnahme vorgenommene Ausheberung des Magens ergibt etwas blutig gefärbte, stark nach Benzin riechende Flüssigkeit. Im Destillat des Mageninhalts Benzin nachgewiesen. Der Magen wird klar gespült. Danach Milch mit 2 Teelöffel Pulv. Liquir. compos. durch die Sonde eingeführt und Kampfer subkutan gegeben. Nach einem warmen Bade mit kühlen Übergießungen wird die Atmung etwas ruhiger, das Kind schläft ein. In den folgenden Stunden erbricht es mehrmals etwas blutige Flüssigkeit. Die Zyanose wird stärker. Die Weite der Pupillen wechselt stark, die Lichtreaktion wird geringer. Das Kind entleert braunroten Urin, der reichlich Eiweiß und viel Sediment enthält, das mikroskopisch größtenteils aus gelblichen, feinkörnigen Massen, vereinzelten geschrumpften roten Blutkörperchen und mehreren granulierten Zylindern besteht. Die Guajakprobe auf Blut fällt stark positiv aus. Der Urin zeigt bei genïgender Verdïnnung das typische Methämoglobinspektrum. Er riecht nicht nach Benzin. Dieses läßt sich auch nicht durch Destillation aus dem Urin gewinnen. Unter zunehmender Zyanose und Atemnot tritt um $3^{3} / 4$ Uhr der Tod ein.

Am nächsten Vormittag wurde im hiesigen pathologischen Institut die Sektion ausgefuhrt. Haut blaß. Totenflecke heller als gewöhnlich. Runzelung der Rachenschleimhaut und der aryepiglottischen Falten (abgelaufenes Ödem?), Blässe der Ösophagusschleimhaut. Glasiger Schleim auf der stark geschwollenen blassen Magenschleimhaut. Diese zum Teil abgelöst. Drei kleine Petechien der hinteren Magenwand. Jejunum fast leer. Der spärliche Dünndarminhalt besteht mikroskopisch fast ansschließlich aus Massen von Zylinderepithelien, die zum Teil zusammenhängende Fetzen bilden. Daneben vereinzelte Erythrocyten. Hyperämie der Schleimhant einzelner Schlingen. Sehr weiche trübe Leber. - Fleckige Hyperämie der Milz. Starke Trübung und Hyperämie der Nieren. Eng kontrahierte Blase mit Spuren eines etwas bräunlichen Urins.

Das Epithel der Bowmanschen Kapseln izt zum Teil desquamiert; in den Kapseln etwas Exsudat. Die Tubuli recti enthalten zum Teil hyaline Zylinder, zum Teil auch degenerierte Erythrocyten. Im Nierenbecken Haufen von Epithelien und Erythrocyten.

Hyperämie der Tracheal- und Bronchialschleimhaut, Aspiration von Mageninhalt bis in die Bronchien des 3. Grades. Umfangreiche Blutungen in das Lungenparenchym und die Pleura, links stärker als rechts. In der linken Brusthöhle etwa 1/4 l rötliche Flüssigkeit, die gelöstes Hämoglobin enthält. Das Sediment der Pleuraflüssigkeit enthält noben roten verhältnismäßig viel weiße polymorphkernige Leukocyten. Gefäßintima, Herzklappen weiß. Keine intravasale Hämoglobinämie.

Sehr starke Hyperämie der grauen Hirnsubstanz. Ödem der Leptomeninx, Ödem des Gehirns (Gewicht $1250 \mathrm{~g}$ ). Starke Blutfüllung des Plexus und der Tela chorioidea. Leicht rötliche, etwas durchscheinende Färbung der nicht vermehrten Ventrikelflüssigkeit.

Hier hat also eine verhältnismäßig kleine Menge von Benzin die tödliche Vergiftung herbeigefuhrt. Im Vordergrunde des klinischen 
und anatomischen Befundes stehen die Lungenerscheinungen: Aspiration von benzinhaltigem Mageninhalt bis in die Bronchien 3. Grades. Zahlreiche Lungenblutungen. Blutige Ergüsse in die beiden Pleuren. Die Angabe, daß das Kind gleich nach dem Trinken gehustet und etwas blutige Flüssigkeit ausgespien, aber nicht erbrochen hat, läßt wohl annehmen, daß es sich sofort beim Trinken verschluckt hat und daß also von Beginn der Vergiftung an das Benzin direkt auf die Lungen einwirken konnte. "Die von vornherein bestehende starke Tachyund Dyspnoe, die Blässe und Zyanose ließen sich auf diese Weise gut als Erstickungserscheinungen erklären. Allerdings ist auch die Möglichkeit gegeben, daß das schwer benommene Kind bei dem späteren mehrmaligen Erbrechen Mageninhalt aspiriert hat.

Als lokale, beim Trinken entstandene. Wirkung ist wohl die Runzelung der blassen Rachenschleimhaut und der aryepiglottischen Falten aufzufassen, die vielleicht auf ein anfängliches akutes Ödem hinweist. Der Magen zeigt außer einigen kleineren Blutungen eine teilweise Abhebung des Epithelbelags. Dementsprechend besteht der spärliche Dünndarminhalt fast ausschließlich aus abgestoßenen Epithelien, denen einige Erythrocyten beigemischt sind. Klinisch batte sich die Magenschädigung durch blutiges Erbrechen zu erkennen gegeben. - Auch hier am Verdauungstraktus handelt es sich um die unmittelbaren lokalen Wirkungen des aufgenommenen Benzins.

Bemerkenswert sind die Urinveränderungen. Bereits intra vitam wurde ein braunroter, eine konzentrierte Methämoglobinlösung darstellender Urin abgeschieden, der reichlich Eiweiß enthielt und in dessen Bodensatz neben zahlreichen Farbstoffkörnchen auch noch stark geschrumpfte, aber deutlich zu erkennende Erythrocyten vorhanden waren. Da weder eine Hämoglobinämie noch Methämoglobinämie bestanden hatte, so ist anzunehmen, daß durch das resorbierte Benzin die Nieren in der Weise geschädigt sind, daß es zu starker Albuminurie und Hämaturie gekommen ist. Die Methämoglobinbildung. dürfte erst extrarenal erfolgt sein. Die durch das Benzin hervorgernfene Nierenschädigung läßt sich auch mikroskopisch deutlich erkennen.

Im Gehirn und seinen Häuten bestand eine starke Hyperämie und ein hochgradiges Ödem, die auf die Wirkung des resorbierten Benzins zu beziehen sind. Der bluthaltige Pleuraerguß zeigte erhebliche Hämolyse. Auch in der Hirnventrikelflüssigkeit schien eine leichte Hämolyse eingetreten zu sein. Eine weitere Umwandlung des Farbstoffes war in der Brusthöhle nicht eingetreten. Das Spektrum gab die für Oxyhämoglobin charakteristischen Linien. Wie bereits 
erwähnt, wies das intravasale Blut keine Veränderungen auf. Die Hämolyse in der Pleura und den Hirnventrikeln konnte also erst nach Abscheidung der Flüssigkeit ans dem Blut entstanden sein.

Der Fall stimmt in bezug auf die Veränderungen am Verdauungstraktus, den Lungen- und Gehirnerscheinungen mit dem iuberein, was bereits wiederholt beschrieben ist. Auch leichte Albuminurie und leichte degenerative Nierenveränderungen sind beobachtet worden. Eine so intensive Nierenblutung, wie sie hier vorgelegen hat, ist uns dagegen aus der Literatur nicht bekannt, ebensowenig die Umwandlung des Hämoglobins in Methämoglobin, die hier im Urin vor sich gegangen war.

Nur selten sind Blutveränderungen beobachtet worden.

Racine erwähnt die eigentiimliche kirschrote Farbe des Blutes und Rotfärbung aller Organe, auch der weißen Hirnsubstanz. Burgl fand hämolysierte Pleuraergüsse und rosarote Färbung der Herzklappen und Sehnenfäden. Beide Autoren messen diesen Blutveränderungen einen wesentlichen Anteil am Vergiftungsbilde zu. Racine verweist auf frühere Versuche von Starkow1), durch die eine hämolytische Wirkung des Benzins festgestellt sei. Nun spricht Starkow zwar von der hämolytischen Wirkung des Benzins, aber nach den angegebenen Formeln der Benzinderivate handelt es sich nicht um Benzin, sondern um Benzol. Klare, der in neuerer Zeit sich die Frage einer etwaigen hämolytischen Wirkung des Benzins vorlegt, hat völlig negative Ergebnisse bei seinen Reagenzglasversuchen, und auch die neuesten Bearbeiter, wie Jaffé und Erben ${ }^{2}$ ), lehnen eine hämolytische Wirkung des Benzins ab.

\section{Fall 2.}

19 jähriges Mädchen hat morgens $8 \mathrm{Uhr}$, um sich das Leben zu nehmen, etwa $75 \mathrm{ccm}$ Benzin and $15 \mathrm{ccm}$. Terpentinöl getrunken. Sie wurde bewußtlos. Der etwa 2 Stunden später hinzugerufene Arzt spülte den Magen gründlich ans. Gegen $12 \mathrm{Uhr}$ kam sie wieder zu Bewußtsein, war aber noch benommen. Um $3 \mathrm{Uhr}$ wurde sie in die Klinik gebracht.

Mittelgroßes Mädchen von mittlerem Ernährungszustande, Gesicht etwas gedunsen, sonst keine abnorme Färbung.

An der Unterseite der Zungenspitze mehrere linsengroße, weißlich belegte Verätzungen. An der Lippenschleimhaut eine punktförmige Blutung. Die hintere Rachenwand erscheint ganz leicht schleierhaft belegt. Pupillen reagieren etwas träge. Puls 100 , sehr weich und wenig gefüllt, Blntdruck $88 \mathrm{~mm} \mathrm{Hg}$ nach Riva-Rocci. Organbefund sonst völlig normal.

1) Virchows Archiv 1871, Bd. 52, S. 464.

2) Erben, Vergiftungen. Wien und Leipzig 1910. 
Die Patientin ist sehr deprimiert, antwortet nur wenig, ist aber klar und macht keinen schwerkranken Eindruck.

Am nächsten Tage ist sie völlig wohl, der Blutdruck ist auf $117 \mathrm{~mm}$ $\mathrm{Hg}$ gestiegen. Der Urin ist dauernd eiweißfrei. Hier hat also die große Menge von $75 \mathrm{ccm}$ Benzin ( $+.15 \mathrm{ccm}$ Terpentin) lediglich eine mehrstündige Bewußtlosigkeit, eine vorübergehende Senkung des Blutdrucks und leichte Verätzungen in der Mundhöhle hervorgerufen. Nach 24 Stunden waren bereits alle Krankheitserscheinungen - außer den Verätzungen wieder geschwunden. Lungenerscheinungen hatten gar nicht vorgelegen. Die Allgemeinvergiftung hatte sich lediglich in Form einer Narkose abgespielt. Das Bild wich stark von dem des vorhergehenden Falles ab.

Wir versuchten uns durch Reagenzglas- und Tierversuche weiteren Aufschluß tiber den Mechanismus der Vergiftung zu verschaffen. Angesichts der widersprechenden Urteile uiber die Einwirkung des Benzins auf das Blut schien es uns erforderlich, über die Frage der hämolytischen Wirkung des Benzins neue Versuche anzustellen. Benzin mischt sich nicht mit Blut oder einer Aufschwemmung gewaschener Blutkörperchen. Man darf sich daher nicht wundern, wenn beim bloßen Zusammenbringen von Blut und Benzin eine Hämolyse nicht eintritt. Bei gründlichem und längerem Schuitteln von Blut und Benzin im Reagenzglas sahen wir dagegen innerhalb von 12-18 Stunden meist eine teilweise Hämolyse eintreten, wenn auch nicht regelmäBig, während die Kontrollen noch unverändert waren. Auch hierbei ist naturgemäß die Einwirkung des Benzins auf die Blutkörperchen nur oberflächlich, da eine eigentliche Mischung doch ausbleibt.

Mit Regelmäßigkeit ist eine kräftige Hämolyse zu erhalten, wenn man eine gesättigte Lösung von Benzin in Rinderserum auf die Blutkörperchen längere Zeit einwirken läßt. Das Serum wird eine halbe Stunde mit etwa der gleichen Menge Benzin stark geschtittelt. Es bildet sich eine Emulsion, aus der sich allmählich wieder eine klare Fluissigkeit absetzt. Dieses klare Benzinserum ließen wir nun im verschlossenen Röhrchen auf Rinderblut oder gewaschene Rinderblutkörperchen unter mehrfachem Umschitteln bei $37^{\circ}$ einwirken. Die Hämolyse tritt nur langsam ein. Nach dreistündiger Einwirkung war in der Mischung von 1-4 Tropfen Benzinserum und 2 ecm gewaschener $10 \%$ iger Rinderblutkörperchen-Aufschwemmung noch keine Veränderung eingetreten. 8 Tropfen Benzinserum und $2 \mathrm{ccm}$ Blutkörperchen-Aufschwemmung ergaben in 3 Stunden leichte, 16 Tropfen Benzinserum und $2 \mathrm{ccm}$ Aufschwemmung stärkere Hämolyse. Nach 6 Stunden war dagegen schon im Glase mit nur 1 Tropfen Benzinserum starke, mit 8 Tropfen völlige Hämolyse eingetreten, während die Kontrollen, die Blutkörperchen-Aufschwemmung und gewöhnliches 
Serum enthielten, noch unverändert waren. Eine hämolytische Wirkung des Benzins ist also mit Sicherheit bei etwas längerer Einwirkung nachzuweisen.

Die hämolytische Beschaffenheit der Pleuraexsudate in den Fällen von Racine und von uns wird daher verständlich. Auch eine intravasale Hämolyse, die wohl bei Racine und Burgl vorgelegen hat, wird man für möglich halten. Aus dem strömenden Blut wird aber das Benzin vielleicht wieder rasch entfernt. Die Einwirkung des Benzins auf die Erythrocyten wird in abgeschlossenen Körperböhlen wohl intensiver sein, besonders wenn, wie es mit den Lungen der Fall ist, Benzin dort aus dem Blute ausgeschieden wird, sich dort also wohl in größeren Konzentrationen ansammeln kann.

Es liegen bereits einige Tierversuche über die Benzinvergiftung vor, die sich allerdings meistens auf die Inhalation des Benzins bezogen. Hierbei waren die Tiere unter zentralen Vergiftungserscheinungen gestorben (Montalti ${ }^{1}$ ), Felix ${ }^{2}$ ), Weißenberg ${ }^{3}$ )).

Die orale Vergiftung mit ihren zur Hauptsache anderen Symptomen war zar Zeit unserer Versuche experimentell nur wenig bearbeitet. Montalti hatte eine toxische Gastroenteritis dabei beobachtet und bezeichnet als tödliche Dosis eine Menge von $7 \mathrm{~g}$ pro Kilogramm. Klare sah bei oraler Vergiftung den Tod unter zerebralen Erscheinungen eintreten. War aus Versehen Benzin in die Lunge gelangt, so traten Lungenblutungen ein. Die Arbeit von Jaffé lag damals noch nicht vor. Jaffé sucht festzustellen, ob die bei der klinischen wie bei der experimentellen Benzinvergiftung nicht seltenen Lungenblutungen nur auf die direkte Einwirkung von aspiriertem Benzin zu beziehen sind, oder ob sie auch infolge Ausscheidung des Benzins aus dem Blnt in die Lungen auftreten können. Er brachte zu diesem Zweck das Benzin direkt in den freigelegten Ösophagus oder Magen ein und beobachtete dabei häufig Lungenblutungen, entschied sich danach dafür, daß auch die Ausscheidung des Benzins in die Lungen zu Blutungen fuluren kann. Gastrointestinale und Blutreränderungen beobachtete Jaffé nicht.

Unsere Versuche galten zunächst ebenfalls der Frage nach der Herkunft der Lungenblutungen, ferner der der Einwirkung auf das Blut. Wenn sie in bezug auf den ersten Punkt auch durch die Arbeit Jaffés überholt sind, so bieten sie doch in anderer Hinsicht manche Eigenheiten, die uns zu ihrer Mitteilung veranlassen.

1) Zit. nach Virchow-Hirschs Jahresbericht 1890, Bd. 1, S. 408.

2) Dt. Vierteljahrsschrift f. offentl. Gesundheitspflege 1872, Bd. 4, S. 266.

3) Dissertat. Wuirzburg 1904. 
Einige orientierende Versuche galten der lokalen Einwirkung des Benzins. Durch die Beobachtungen von Pürchbauer'), Levy ${ }^{2}$ ), Hörrmann ${ }^{3}$ ), Sehrwald ${ }^{4}$ ) ist nachgewiesen, daß Benzin, dessen Verdunstung verhindert wird, lokale Nekrosen auf der Haut hervorrufen kann. In den Hohlräumen des Körpers ist nun das Benzin ebenfalls am Verdunsten gehindert, eine Ätzwirkung daher naheliegend. Um diese $z u$ prufen, wurden Organstückchen gesunder Tiere für 24 Stunden in Benzin und andere zur Kontrolle in NaCl-Lösung eingelegt. Die in Kochsalz aufbewahrte Magenwand zeigte keine gröberen Veränderungen, dagegen war im Benzinversuch das Magenepithel gröBtenteils in eine gallertige leicht abstreifbare Masse umgewandelt, die mikroskopisch aus Massen einzelner oder in Verbänden liegender, ziemlich gut erhaltener Epithelien bestand.

Ein in Benzin aufbewahrtes Lungenstiuck sieht - besonders in seinen Randpartien - wesentlich heller aus als das Kontrollstiick. Der leicht ausdriickbare Saft enthält sehr große Mengen von Alveolar- und Bronchialepithelien. Der aus der Kontrollunge ausgedriickte Saft enthält zwar reichlich Erythrocyten, aber nur vereinzelte Epithelien. Das Benzin hat danach die Fähigkeit, die Gewebe so zu verändern, daß die Epithelien sich leicht ablösen. Sie selbst werden dabei nur in geringerem Maße verändert. Es macht den Eindruck, als wenn die Kittsubstanz zwischen den Epithelien der Auflösung verfällt. Die lokale schädigende Wirkung wird also durch diese Versuche bestätigt.

Die folgenden Versuche galten der stomachalen Vergiftung.

\section{Versuch 1.}

Einem Kaninchen, das 4 Tage gehungert hat, wurden $20 \mathrm{ccm}$ Benzin durch die Schlundsonde in den Magen eingeführt. Es starb nach 6 Tagen. Die Schleimhaut des Magens und zum Teil auch des Dünndarms war leicht abstreifbar, die Leber stark hyperämisch, đie Niere getrübt; die Lungen wiesen mehrfache Blutungen auf, der Blasenurin enthielt reichlich Eiweiß, Zelltrümmer und einzelne granulierte Zylinder.

Versuch 2.

Ein Hund von $1 \mathrm{~kg}$ Gewicht, der nach $1 \frac{1}{2}$ tägigem Hungern $25 \mathrm{ccm}$ Benzin mit der Schlundsonde erhalten hatte, starb nach 36 Stunden. Un-

1) Minch. med. Wochenschrift 1910, Nr. 42.

2) Ebenda 1911, Nr. 6.

3) Ebenda 1911, Nr. 21.

4) Deutsche med. Wochenschrift 1913, Nr. 7 . 
mittelbar nach der Einführung des Benzins war sein Gang taumelnd, in den nächsten Stunden war das Tier sehr unruhig.

Auch hier zeigte sich wieder eine erhebliche Desquamation der Magenschleimhaut, Hyperămie der Leber, Nierentrübung, einzelne Lungenblutungen. Die Luftwege waren frei von Aspirationen. Der Urin enthielt etwas Eiweiß und mikroskopisch Haufen von Epithelien.

In beiden Versuchen zeigte sich also die schädigende Wirkung des Benzins auf Magen, Nieren und Lungen.

\section{Versuch 3.}

Während der Hund des Versuchs 2 bereits nach $1^{1 / 2}$ Tagen starb, blieb ein gleieh schwerer Hund, der nach 2 tägigem Hungern fast die doppelte Benzimmenge (40 cem per Schlundsonde) erhalten hatte, fast 3 Wochen am Leben. Auch hier trat nach der Einführung des Benzins der taumelnde Gang, später die große Unruhe des Tieres anf. Der Urin enthielt anfangs wenig, später mehr Eiweiß, Zelltrümmer, später auch Leukocyten und einzelne granulierte Zylinder. Die Sektion wurde nicht gemacht.

Eine unverhältnismäßig große Dose hatte bei diesem Tier also erst nach längerer Zeit den Tod herbeigefuihrt. Schwere akute Erscheinungen hatten gefehlt. Mit Riicksicht auf die besondere Empfindlichkeit der Kinder gegenuber der Benzinvergiftung wurden zu den nächsten Versuchen drei junge 3 Wochen alte Hunde des gleichen Wurfs benutzt. Sie erhielten - ebenfalls durch die Schlundsonde 15-25 ccm Benzin. Auch hier blieben bei dem ersten dieser Hunde schwere akute Vergiftungserscheinungen aus. Er wurde nach 4 Tagen getötet. Die beiden anderen Tiere, die ebenfalls ohne schwere akute Erscheinungen geblieben waren, wurden deshalb schon nach 24 Stunden getötet, um festzustellen, welche Veränderungen kurze Zeit nach der Vergiftung beständen.

\section{Versuch 4.}

3 Wochen alter Hund, 4 Stunden gehungert, dann $15 \mathrm{ccm}$ Benzin durch Schlundsonde. Erbricht einen Teil des Benzins, 11 Stunden später an die Mutter gelegt. Bleibt dauernd munter. Urin eiweißfrei. Nach 4 Tagen entblutet. Organe ohne Besonderheiten, ebenso der Magen- und Koloninhalt bei mikroskopischer Untersuchung. Der Duodenalinhalt enthält dagegen Zylinderepithelien und einzelne Pigmenthäufchen.

Hier ist infolge des frühzeitigen Erbrechens wohl nur wenig Benzin zur Resorption gelangt, schwerere Vergiftungserscheinungen haben dementsprechend gefehlt. Die Schädignng des Verdauungstraktus zeigt sich im Befunde des Duodenalinhalts. 


\section{Versuch 5 .}

Hund, 3 Wochen alt. Nach 24 stündigem Hungern $25 \mathrm{ccm}$ Benzin per Schlundsonde. Ein wenig davon wird regurgitiert und vielleicht aspiriert. Der Hund ist danach sehr matt, fällt um, bekommt Krämpfe (Erstickung?). Am nächsten Morgen ist er völlig munter. Nach 24 Stunden wird er entblutet. Der Magen enthält reichliche dicke, klare Schleimmassen, die stellenweise gelblich und rötlich gefürbt sind. Mikroskopisch ist der Schleim ganz von Erythrocyten durchsetzt. Daneben an einzelnen Stellen gelbe Pigmentmassen. Keine freien Epithelien. Darm äußerlich ohne Besonderheiten. Auch mikroskopisch weist der Magen, die Duodenalund Dickdarmschleimhaut nichts Abnormes auf.

Der Dünndarm enthält galligen Schleim mit Epithelien. Die Niere läßt mikroskopisch Desquamation im Nierenbecken, hyaline Zylinder in den Sammelröhrchen und Tubuli recti, einzelne Erythrocyten in den Tubuli recti und dem Spalt der Glomeruli erkennen. In der rechten Lunge reichlich größere Blutungen, in der linken ein Herd. Mikroskopisches Bild: die Alveolen sind dicht mit Erythrocyten, zum Teil auch mit hyalinen Massen gefüllt. In der Blase flockig getrübter Urin, der Massen von großen granulierten Zylindern mit vereinzelten aufgelagerten Erythrocyten, ferner reichlich freie Erythrocyten, einige Häufchen Epithelzellen and viel Eiweiß enthält.

Die Schädigung der Magenwand ist hier gering, vielleicht infolge der sehr starken Schleimabsonderung. Die Niere ist deutlich geschädigt; dem entsprechen die starken Urinveränderungen. Die Lungenblutungen sind hier vielleicht durch Aspiration entstanden.

\section{Versuch 6.}

Hund, 3 Wochen alt. Nach 24 stündigem Hungern $20 \mathrm{ccm}$ Benzin durch die Schlundsonde. Er schreit dabei stark, verschluckt sich aber nicht: der Gang ist zunächst ewas wackelig, am nächsten Tage aber ist das Tier wieder völlig munter. Entblutet nach 24 Stunden. Magen und Dünndarm sind mit bräunlich-blutigem Schleim gefüllt, mikroskopisch sind Leukocyten, degenerierte Epithelien, ausgefallener Blutfarbstoff zu erkennen. Intakte rote Blutkörperchen sind nicht mehr vorhanden. Der Dickdarm ist mit einer teerartigen Masse gefulltt, in der mikroskopisch viele Zellreste nachgewiesen sind und die chemisch, anch nach starker Verdtinnung, noch ausgesprochene Blutreaktion gibt (Guajak- und Aloinprobe). Mikroskopiseh zeigt die Magenschleimhaut (ein Teil des Magens war unaufgeschnitten gehärtet) Auflagerungen von Blut, Epithelien und Zelltrümmern. Die Epithelien der Mucosa sind zum Teil gut erhalten, zum Teil schlecht gefärbt. Viele sind desquamiert. Duodenum, Pankreas, Dickdarm sind mikroskopisch normal.

Der Blasenurin enthält etwas Eiweiß und eine mäßige Menge von Erythrocyten und granulierten Zylindern. Die Niere läßt Exsudation in die Glomeruluskapseln erkennen. Feinkörnige Fettablagernng im basalen Teil der Epithelien der Tubuli contorti. Die Tubuli recti zeigen an einzelnen Stellen Epitheldesquamation und Detritus in Lumen. 
Die rechte Lunge enthält einen kirschgroßen hämorrhagischen Herd, die linke Lunge ist normal. Mikroskopisch bietet der Herd dasselbe Bild wie im vorigen Präparat.

Hier sind die Magenschädigungen erheblicher. Die Niere ist wieder betroffen. Ein Blutungsherd in der Lunge ist bei diesem Tier vorhanden, das, soweit zu beobachten, nicht aspiriert hatte.

Bei den sezierten Tieren, von dem nur ganz leicht vergifteten Tier Versuch 4 abgesehen, waren also lokale Veränderungen im Verdauungstrakt vorhanden, allerdings von wechselnder Stärke, ebenso Nierenschädigungen. Alle 4 Tiere wiesen ferner Lungenblutungen anf, die in Versuch 5 wohl auf Aspiration von flüssigem Benzin zu beziehen waren, während bei den anderen Tieren Aspirationen nicht beobachtet wurden.

Die Jaffé schen Versuche, wie auch diese drei Tierversuche, machen also das Auftreten von Lungenblutungen infolge von Benzinausscheidung wahrscheinlich. Immerhin besteht vielleicht die Möglichkeit, daß das schon bei Körpertemperatur stark verdampfende Benzin gelegentlich in den Ösophagus gelangt und rom Rachen aus in die Lungen inspiriert wird. Es könnte auf die Weise doch wieder eine unmittelbare Benzinwirkung zustande kommen. Daß bei Inhalation von Benzin Lungenblutungen auftreten können, hat Dorendorf beobachtet.

Auffallend ist, daß die drei jungen Hunde eine viel geringere Empfindlichkeit gegen Benzin gezeigt haben, als Kinder. Obwohl sie verhältnismäßig sehr viel mehr Benzin bekommen hatten, als bei Kindern zur tödlichen Vergiftung ausgereicht hatte, waren sie ohne schwerere Krankheitserscheinungen geblieben. Es ist oben bereits hervorgehoben worden, daß Erwachsene oft unverhältnismäßig viel größere Mengen von Benzin vertragen, als Kinder. Die rasch schon nach kleinen Mengen Benzin eintretenden Todesfälle bei Kindern sind wohl auf Aspiration zu beziehen. In dem Augenblick, wo das Benzin in die Mund- und Rachenhöhle gelangt, wird es zu verdunsten beginnen, die Luft verdrängen und so Atemnot und unregelmäßige tiefe Inspirationen hervorrufen, bei denen leicht auch fliussiges Benzin mit aspiriert werden kann. Der Schreck über das brennende und ïbelriechende Getränk mag ebenfalls leicht zum Verschlucken führen. Gelangt das Benzin ausschließlich in den Magen, so scheint es weit weniger gefährlich zu sein. Klare und Jaffé kommen zu ähnlichen Schlüssen.

Es war in diesen Versuchen nirgends zur Bildung von Pleuraergiussen gekommen. Da diese und die in ihnen aufgetretene Hämolyse in unserem klinischen Falle 1 so im Vordergrund standen, so wurde 
bei weiteren Versuchen das Benzin unmittelbar in die Pleurahöhle gebracht, um die lokalen Veränderungen untersuchen zu können.

\section{Versuch 7 .}

Einem mittelgroßen Kaninchen werden $20 \mathrm{ccm}$ Benzin in die rechte Pleurahöhle injiziert. Das Tier stirbt sofort danach, anscheinend an Erstickung (Krämpfe, schnappende Atmung). Die rechte Brusthöhle enthält große Klumpen geronnenes Blut, daneben etwas ungeronnenes, zum Teil bämolysiertes Blut und etwas Benzin. Die Lungen, Bronchien und die Trachea enthalten geronnenes Blut. Wahrscheinlich ist bei der Panktion die Lunge angerissen worden. Der Versuch zeigt jedenfalls, daß auch in der Brusthöhle unter der Benzineinwirkung eine Hämolyse stattfinden kann.

\section{Versuch 8.}

Ein mittelgroßes Kaninchen erhält $10 \mathrm{ccm}$ Benzin in die rechte Brusthöhle. Die Einspritzung wird reaktionslos vertragen. Das Tier ist nur etwas stumpf danach. In der Nacht stirbt es. Sektion: Von der eröffneten Bauchhöhle aus erkennt man, daß in beiden Brusthöhlen sich Flüssigkeit befindet. Diese wird unter Vermeidung jedes nachträglichen Bluteinflusses in die Brusthöhle mit der Spritze angesaugt. Die rechte Brusthöhle enthält $7 \mathrm{ccm}$ Flüssigkeit, die sich aus $2 \mathrm{ccm}$ eines sehr stark hämoglobinhaltigen Exsudats und $5 \mathrm{ccm}$ Benzin zusammensetzt. Die linke Brusthöhle enthält 5 ccm rotes hämolysiertes Exșudat. Die Lungen sind äußerlich unverletzt, sie enthalten mehrfache Hämorrhagien. Mikroskopisch sind die Alveolen mit Erythrocyten gefïllt, die zum Teil intakt sind, zum Teil einen Übergang in homogene Massen zeigen. Andere Alveolen haben völlig homogenen glasigen Inhalt. Die Alveolarepithelien sind großenteils abgestoßen, sie enthalten vielfache gelbbraune Pigmentkörnchen. Auch die Alveolarsepten sind zum Teil degeneriert. Die Pleura ist an manchen Stellen wie aufgefasert, der Endothelbelag arrodiert. Die Bronchien sind großenteils frei, zum Teil enthalten sie Erythrocyten oder homogene glasige Massen. Das Mediastinum ist gallertig gequollen. Im Herzbeutel einige Tropfen Flüssigkeit, die ebenfalls freies Hämoglobin enthält. Das Herzblut ist nicht hämolysiert. Der Blasenurin enthält ein wenig Eiweiß.

Hier hatte also bei längerer lokaler Einwirkung das Benzin erhebliche Veränderungen hervorgerufen. Es war augenscheinlich von der rechten Brusthöhle auch in die linke und das Perikard diffundiert und hatte uiberall zur Bildung blutiger Exsudate und zur Hämolyse geführt. Das Bindegewebe des Mediastinums war gallertig gequollen. Auch in die Lungen selbst war das Benzin eingedrungen und hatte dort zu Hämorrhagien, Hämolyse und Abstoßung der Alveolarepithelien geführt.

\section{Versuch 9 .}

Mittelgroßes Kaninchen. $\mathbf{5}$ cem Benzin in die rechte Pleurahöhle. Sofort danach schnarchende Atmung. Das Tier stirbt nach 8 Minuten. Sektion erst nach einigen Stunden. 
Die Injektion ist anscheinend in die Lunge erfolgt, hat hier zu großen Blutungen geführt, auch die Trachea und die Bronchien sind mit Blut und hämoglobinhaltiger Flüssigkeit gefüllt, die noch deutlich nach Benzin riecht. In beiden Brusthöhlen und in der Bauchhöhle einige Kubikzentimeter hämoglobinämische Flüssigkeit. Intravasales Blut gelöst.

Also auch hier Hämolyse an den Stellen, wo das Benzin unmittelbar einwirkte. Die Hämolyse ist vielleicht erst post mortem erfolgt.

\section{Versuch 10.}

Mittelgroßes Kaninchen, $5 \mathrm{ccm}$ Benzin in die rechte Brusthöhle injiziert. Keine Dyspnoe danach. Das Tier stirbt in der Nacht. In der Bauchhöhle $1 \mathrm{ccm}$ leicht hämolysierte Flüssigkeit. In beiden Pleuren je einige Kubikzentimeter Flüssigkeit. In die rechte Brusthöble läuft bei der Eröffnung etwas Blut, die stark blutige Flüssigkeit der linken Brusthöhle gerinnt bei der Herausnahme im ganzen; über eine etwaige Hämolyse ist also kein Urteil zu gewinnen. In beiden Brusthöhlen etwas Benzin. Die rechte Lnnge weist eine Stichverletzung anf. In beiden Lungen kleine Hämorrhagien, die mikroskopisch das gleiche Bild wie im vorigen Fall darbieten, also auch eine partielle Hämolyse erkennen lassen.

Aus den Versuchen 7-10 geht also hervor, daß Benzin bei direkter Einwirkung auf die Pleura Blutungen und Hämolyse erzengt. Das Benzin diffundiert dabei durch das Mediastinum, das dabei glasig gequollene Beschaffenheit annehmen kann, in die andere Pleurahöhle und unter Umständen auch in den Herzbeutel und die Bauchhöhle und erzeugt dort die gleichen Veränderungen. Auch in die Lungen kann es durch Diffusion gelangen und Bluteintritt und Hämolyse hervorrufen.

Als Ergebnis der Tierversuche und der Beobachtungen bei menschlichen Vergiftungen läßt sich danach sagen: Das Benzin vermag dort, wo es längere Zeit einwirkt, lokale, sich besonders in Epithelablösung und Blutaustritt äußernde Gewebsschädigung und Hämolyse des ausgetretenen Blutes hervorzurufen. Die klinischen Beobachtungen machen es wahrscheinlich, daß gelegentlich auch eine intravasale Hämolyse eintreten kann. Es werden weiter regelmäßig die Nieren geschädigt; auch dort kann es, wie unser klinischer Fall erweist, zu stärkerem Blutaustritt kommen. Der Farbstoff des ausgetretenen Blutes ist hier nachträglich in Methämoglobin umgewandelt worden. Weiter wird, wie aus den klinischen und anatomischen Beobachtungen hervorgeht, das Zentralnervensystem geschädigt. Die häufigen Lungenblutungen beruhen - wenigstens bei Kindern.- wohl großenteils auf der unmittelbaren Wirkung des aspirierten Benzins, können aber nach den Tierversuchen - ähnlich wie die Nierenschädigungen - auch durch 
resorbiertes und in den Lungen ausgeschiedenes Benzin hervorgerufen werden. Die raschen Todesfälle der Kinder sind, wie wir in Übereinstimmung mit Klare und Jaffé annehmen, großenteils wohl auf Aspiration von Benzin zu beziehen. Wenn die Vergiftung lediglich durch Resorption vom Magen aus zustande kommt - das ist wohl meist bei Erwachsenen und auch bei manchen Kindern der Fall -, so tritt sie langsamer ein und ist gutartiger. Es stehen dann die zerebralen Erscheinungen im Vordergrunde, Lungenerscheinungen können aber ebenfalls auftreten. 